\title{
PRÁCTICAS PEDAGÓGICAS EN AULA MULTIGRADO: PRINCIPALES DESAFÍOS SOCIOEDUCATIVOS EN CHILE
}

Pamela Andrea Freire-Contreras ${ }^{1}$

Gabriela Ninoska Llanquín-YaupiII

Dalesca Estefanía Neira-Toledo III

Elisabet Noemí Queupumil-Huaiquinao ${ }^{\mathrm{IV}}$

Lidia Abigail Riquelme Hueichaleov

Katerin Elizabeth Arias-Ortega ${ }^{\text {VI }}$

I, II, III, IV, v, vi Universidad Católica de Temuco (UCT), Temuco, Chile; karias@uct.cl

\section{Resumen}

El artículo presenta resultados de investigación sobre la percepción de profesores de aulas multigrado respecto de los principales desafíos socioeducativos que enfrentan en sus prácticas pedagógicas, en una escuela situada en comunidad mapuche de La Araucanía, Chile. La metodología utilizada es cualitativa. Los principales resultados dan cuenta que los profesores que se desempeñan en escuelas multigrados deben atender de forma simultánea la diversidad social, cultural, lingüística y etaria que presentan los estudiantes, pese a no tener la formación profesional para ello. Esta realidad se complejiza, si se consideran las características del currículum escolar monocultural que no responde a la modalidad de escuelas multigrado, ni al contexto donde están insertas dichas escuelas.

PRÁCTICAS PEDAGÓGICAS • CLASE MULTISERIADA • ENSEÑANZA • APRENDIZAJE

\section{PRÁTICAS PEDAGÓGICAS EM TURMAS MULTINÍVEL: PRINCIPAIS DESAFIOS SOCIOEDUCACIONAIS NO CHILE}

\section{Resumo}

O artigo apresenta resultados de pesquisa sobre a percepção de professores de turmas multinível no tocante aos principais desafios socioeducativos enfrentados nas suas práticas pedagógicas em uma escola situada em uma comunidade mapuche de La Araucanía, Chile. A metodologia utilizada é qualitativa. Os principais resultados evidenciam que os professores que lecionam em escolas multinível devem atender simultaneamente a diversidade social, cultural, linguística e etária dos alunos, embora não tenham formação profissional para isso. Essa realidade se torna ainda mais complexa se forem consideradas as características do currículo escolar monocultural que não corresponde à modalidade das escolas multinível, nem ao contexto em que tais escolas estão inseridas.

PRÁTICAS PEDAGÓGICAS • CLASSE MULTISSERIADA • ENSINO • APRENDIZAGEM 


\section{PEDAGOGICAL PRACTICES IN MULTIGRADE CLASSROOM: MAIN SOCIO- -EDUCATIONAL CHALLENGES IN CHILE}

\section{Abstract}

The article presents research results on the perception of teachers of multigrade classrooms regarding the main socio-educational challenges they face in their pedagogical practices, in a school in La Araucanía, Chile. The methodology used is qualitative. The main results show that teachers who work in multigrade schools must simultaneously attend to the social, cultural, linguistic and age diversity presented by the students, despite not having the professional training to do so. This reality becomes more complex, considering the characteristics of the monocultural school curriculum that does not respond to the multigrade school modality, or the context where these schools are inserted.

TEACHING PRACTICES • MULTI-SERIES CLASS • TEACHING • LEARNING

\section{PRATIQUES PÉDAGOGIQUES DANS DES CLASSES MULTI-NIVEAUX AU CHILI : PRINCIPAUX DÉFIS SOCIO-ÉDUCATIFS}

\section{Résumé}

L'article présente les résultats d'une recherche sur la perception d'enseignants de classes multi-niveaux d'une école située dans une communauté mapuche de La Araucanía au Chili. Elle concerne les principaux défis socio-éducatifs auxquels ils sont confrontés dans leurs pratiques pédagogiques. La méthodologie utilisée est de nature qualitative. Les résultats principaux indiquent que les professeurs dans des écoles multi-niveaux doivent répondre à la fois à la diversité sociale, culturelle, linguistique et d'âge des élèves, sans pour autant avoir reçu une formation professionnelle dans ce domaine. Cette réalité devient encore plus complexe si l'on prend en compte les caractéristiques du programme scolaire monoculturel qui n'est pas adapté aux classes multi-niveaux ni au contexte dans lequel ces écoles s'insèrent.

PRATIQUES PÉDAGOGIQUES • CLASSE MULTI-SÉRIES • ENSEIGNEMENT • APPRENTISSAGE 

generaciones adquirir habilidades y conocimientos de acuerdo al marco social y cultural de la sociedad hegemónica. Esto, para desenvolverse eficazmente dentro de los marcos productivos, sociales, culturales, aportando al desarrollo económico de su sociedad (Vera et al., 2012). La educación formal implica un desafío para el profesor quién debe asumir la responsabilidad de formar a los nuevos ciudadanos en base a los saberes y conocimientos educativos hegemónicos que han sido incorporados al currículum escolar. Así, el profesor debe ser capaz de propiciar en cada uno de los estudiantes el desarrollo de competencias, conocimientos y el logro de los objetivos de aprendizaje, según lo estipulado en el Marco Curricular Nacional chileno. Lo anterior, independientemente de las características individuales, sociales, culturales y lingüísticas propias del individuo, ya que en el sistema escolar es asumido como un sujeto homogéneo, invisibilizando su diversidad social y cultural en el aula. El sistema escolar chileno, históricamente, se ha caracterizado por ofrecer a las nuevas generaciones una educación monocultural y monolingüe única, sin importar sus especificidades y la diversidad social y cultural que coexisten en los espacios educativos (Arias-Ortega, 2019). Esta realidad da cuenta de la complejidad que implica el desarrollo de las prácticas pedagógicas, en el que el profesor debe asegurar el éxito escolar y educativo de todos los estudiantes.

El sistema educativo escolar chileno se caracteriza por impartir educación formal desde los 5 hasta los 18 años de edad, en los siguientes niveles educativos: 1) Educación Parvularia, que favorece de manera oportuna y pertinente el desarrollo integral y la adquisición de aprendizajes relevantes y significativos en los niños (Agencia de Calidad de la Educación, 2018);2) Educación Básica, que entrega a los estudiantes aprendizajes que conducen a su autonomía, permitiéndoles adquirir las herramientas y habilidades necesarias para participar en la sociedad. Este nivel educativo es obligatorio para todos los niños en edad escolar (Agencia de Calidad de la Educación, 2018); 3) Educación Media, que procura que cada alumno expanda y profundice su formación general y desarrolle los conocimientos, habilidades y actitudes necesarios para ejercer una ciudadanía activa e integrarse a la sociedad de manera productiva (Ley n. 20370, 2009); 4) Educación Superior, que tiene como objetivo la preparación y formación del estudiante en un nivel avanzado en las ciencias, las artes, las humanidades y las tecnologías, y en el campo profesional y técnico. Este nivel educativo no es obligatorio para los estudiantes chilenos (Ley n. 20370, 2009). No obstante, en las últimas décadas ha aumentado el ingreso de estudiantes de estratos sociales más bajos, puesto que se han dado mayores oportunidades de ingreso a través del Sistema Nacional de Financiamiento Estudiantil, garantizando por medio de créditos y becas el apoyo económico, para financiar total o parcialmente los aranceles de los jóvenes que lo necesitan (Arias-Ortega y Quintriqueo-Millán, 2020).

En Chile el componente geográfico y territorial caracterizado por largas distancias generó la instalación de escuelas multigrado en espacios geográficos aislados y con difícil acceso, para ofrecer una educación a los niños que allí habitan. Así, en el territorio nacional emergen y se instalan escuelas multigrados y unidocentes. Las escuelas multigrado se caracterizan por ser atendidas por uno o más profesores que desarrollan los procesos de enseñanza y aprendizaje en cursos combinados en la misma sala de clases (Estrada, 2015). Este tipo de escuelas se encuentran generalmente ubicadas en sectores rurales con comunidades indígenas, donde se considera que no hay suficientes alumnos para que sea costeable conformar un grupo para cada grado. Mientras que las escuelas unidocentes son aquellas que albergan a primer y segundo ciclo de enseñanza básica en una misma sala de clases, y que generalmente, se caracterizan por tener una matrícula menor a treinta estudiantes, los que son atendidos por un solo profesor (Chávez \& García, 2013). En el caso particular de La Araucanía, los datos del Ministerio de Educación de Chile 
nos permiten constatar que existe un total de 3.524 establecimientos rurales, de los cuales el 51,8\% corresponde a las denominadas escuelas multigrado (Agencia de Calidad de la Educación, 2018). Estas escuelas se encuentran situadas mayoritariamente en zonas apartadas del territorio urbano y atienden, principalmente, a las poblaciones rurales e indígenas quienes presentan contextos territoriales con mayores índices de vulnerabilidad social a nivel país (Rodríguez, 2004). Asimismo, son las escuelas que históricamente han obtenido los más bajos resultados en las evaluaciones estandarizadas a nivel país (Arteaga Martinez et al., 2017).

La revisión de la literatura respecto a experiencias de escuelas multigrado, sus características y funcionamiento tanto a nivel internacional, nacional y local, en países como México, Brasil, Ecuador y Chile, nos ha permitido evidenciar que en general las escuelas rurales multigrado y unidocente presentan algunas problemáticas que limitan los procesos de enseñanza y aprendizaje, tales como: 1) las condiciones materiales en que operan las escuelas se caracterizan por una mala infraestructura (Santos, 2017; Castillo-Miranda et al., 2017); 2) la irregular e inadecuada utilización del tiempo escolar, producto de la distancia entre el hogar del estudiante y su establecimiento (Ames, 2004); 3) la escasa organización administrativa de las escuelas y su aislamiento de sectores urbanos, lo que impide establecer redes de apoyo y colaboración socioeducativa con otros centros o instituciones gubernamentales (Arteaga Martinez et al., 2017); 4) la inadecuada distribución del tiempo en las tareas y la planificación de las clases por parte de los profesores, quienes deben atender distintos niveles educativos en la misma sala de clases (Vera \& Peña, 2016; Santos, 2017); y 5) la educación rural ha sido históricamente marginada en la construcción de políticas públicas y tratada como una política compensatoria (Santos, 2017). De este modo, la educación ofrecida se caracteriza por discursos, perfiles y currículos escolares descontextualizados de la realidad territorial en el que se imparte la enseñanza, desviándose de las necesidades locales y regionales, incidiendo negativamente en las prácticas pedagógicas y afectando el éxito escolar y educativo de los estudiantes (Zambrano, 2017).

En Chile, históricamente las prácticas pedagógicas en escuelas rurales se han caracterizado por ser monoculturales y descontextualizadas de los saberes y conocimientos propios de los estudiantes (Arias-Ortega, 2019). Hipotéticamente, esto podría explicar los bajos resultados obtenidos en las evaluaciones estandarizadas que afectan el éxito escolar y educativo de los estudiantes. El éxito escolar referido a la adquisición y dominio de conocimientos, saberes y competencias propuestos por los programas escolares que son certificados en la educación escolar. Mientras tanto, el éxito educativo refiere al desarrollo global y óptimo de la persona, considerando las dimensiones de su ser a nivel cognitivo, espiritual, afectivo, físico y social, lo que implica considerar el medio social y cultural en el que se desarrolla la educación escolar (Arias-Ortega, 2019).

En consecuencia, la revisión del estado del arte nos ha permitido constatar que existen estudios asociados a las escuelas multigrado y unidocente tanto a nivel internacional, nacional como local. Dichos estudios se han focalizado, principalmente, en comprender cómo es el funcionamiento de estos establecimientos en el marco de su infraestructura y recursos materiales. Sin embargo, constatamos que se carece de estudios que aborden la realidad que se vive en dichas escuelas desde las percepciones de los propios sujetos que desarrollan los procesos de enseñanza y aprendizaje, es decir, desde el profesorado. Asimismo, constatamos que se carece de estudios en La Araucanía que aborden dicha realidad. Esto es complejo, si consideramos el alto porcentaje de este tipo de escuelas en el territorio. De esta manera la pregunta de investigación que guía el estudio es: ¿Cuáles son los principales desafíos socioeducativos que enfrentan los profesores que se desempeñan en aula multigrado en contexto rural en La Araucanía?

El objetivo del artículo es exponer los resultados de la investigación que indaga sobre los desafíos y factores socioeducativos que enfrentan los profesores de aula multigrado en una escuela rural de La Araucanía, Chile. 


\section{Experiencias de escuelas multigrado a nivel internacional}

Las escuelas multigrado se caracterizan porque en el proceso de enseñanza y aprendizaje interactúan distintos niveles educativos entre sí. Esto genera que la enseñanza se desarrolle en un ambiente particular y en directa relación entre el profesor y sus estudiantes, constituyendo experiencias de aprendizaje únicas. Pero a la vez, implica una colaboración directa entre pares, independientemente del nivel escolar y el rango etario, puesto que los estudiantes al compartir el mismo espacio educativo están en constante interacción. Lo anterior favorece el desarrollo del sentido ético, moral y actitudinal en los niños, sustentados en el respeto, reciprocidad y colaboración entre pares, durante su proceso de enseñanza y aprendizaje. Desde la perspectiva de la teoría sociocultural de Vigotsky (1997), la educación fracasa si no toma en consideración las diversas interconexiones del niño con el ambiente y/o si está alejada de su vida real, lo que en cierta forma dificulta la adecuada adquisición de aprendizajes de los estudiantes, al recibir enseñanzas descontextualizadas (Luria et al., 2004). De este modo, el enfoque sociocultural de Vigotsky (1997), nutre la perspectiva de las prácticas pedagógicas que se observan en el contexto rural en un aula multigrado, pues es en este contexto donde se desarrollan las interacciones entre la escuela y la comunidad, lo que favorece al desarrollo integral de los estudiantes, teniendo en consideración su propio marco de referencia.

Las experiencias de prácticas pedagógicas en el contexto de aulas multigrados a nivel internacional, dan cuenta que este tipo de escuelas está presente en diferentes países, tal es el caso de Finlandia y España (Boix, 2011). En dichos ámbitos, se han desarrollado investigaciones que buscan problematizar y comprender el funcionamiento de las prácticas pedagógicas en aulas multigrado. En los resultados de estudio se han identificado las principales limitaciones y posibilidades que emergen en estas escuelas y que podrían contribuir en una toma de decisiones oportunas. Esto para asegurar procesos de enseñanza y aprendizaje de alta calidad, para los niños y familias que asisten a escuelas multigrado. Las experiencias de Finlandia dan cuenta que las escuelas multigrado y sus políticas públicas permiten que todos los niños, independientemente de su contexto socioeconómico o territorial, puedan tener acceso igualitario y gratuito a escuelas de calidad (Juárez, 2012). Este tipo de escuelas multigrado es altamente efectivo en función de: 1) se ven reforzadas por las políticas públicas que generan una mayor equidad e igualdad social en cuestiones de ingresos y bienestar social, el Estado invierte elevados recursos económicos para mejorar su funcionamiento (Niemi, 2015); 2) las prácticas pedagógicas se caracterizan por incorporar en los procesos de enseñanza y aprendizaje una alta flexibilidad, para atender a la diversidad cultural del alumnado (Bustos, 2014); 3) se han instalado bibliotecas en autobuses que visitan las escuelas y comunidades más remotas al menos una vez al mes, permitiendo ofrecer herramientas y oportunidades de aprendizaje y acercamiento a la lectura a todos los estudiantes (Juárez, 2012). Sin embargo, aun cuando se ha implementado una serie de políticas públicas para apoyar los procesos de enseñanza y aprendizaje en este tipo de establecimientos, aún persisten problemáticas como: 1) este tipo de escuelas en general carecen de los recursos económicos suficientes para dotar de una buena biblioteca a toda y cada una de las escuelas del país; y 2) los profesores reconocen que aún necesitan seguir profundizando y capacitándose en herramientas didácticas y pedagógicas para desempeñarse en escuelas multigrado (Juárez, 2012).

Lo anterior es coherente con los resultados de investigación sobre las prácticas pedagógicas del profesorado en escuelas multigrado del contexto español, en los que se identifican factores que benefician el quehacer de la escuela multigrado, intentando asegurar permanentemente su calidad educativa. Los resultados de investigación de Quilez y Vázquez (2012); Gallardo (2011) y Pérez et al. (2018) destacan que existe: 1) mayor conexión entre las culturas de los estudiantes y de los adultos, ya que al ser un grupo reducido permite trabajar en profundidad las tradiciones y costumbres de la población que atiende (Quilez \& Vázquez, 2012); 2) permiten responder a las necesidades de los estudiantes, pues 
existe una relación individual que establecen los docentes con el alumnado, donde consideran sus raíces culturales, entorno y características personales (Gallardo, 2011); 3) obtienen mejores calificaciones que los contextos urbanos, debido a que tienen baja cantidad de estudiantes, lo que facilita mayor personalización de la enseñanza (Pérez et al., 2018). No obstante, estas escuelas no se encuentran exentas de problemas como: 1) existe una baja cantidad de población en la zona rural y disminución de estudiantes en edad escolar, debido a la escasa oferta laboral, lo que genera que las familias emigren a contextos urbanos (Gallardo, 2011); 2) se carece de recursos económicos, lo que limita su acceso a materiales pedagógicos e infraestructura adecuada (Gallardo, 2011); y 3) la formación de los profesores no siempre da respuesta a las particularidades de este tipo de establecimiento, quedando a su voluntad el desarrollo profesional (Pérez et al., 2018).

En América Latina también constatamos experiencias de escuelas multigrado en el sistema escolar. Por ejemplo, en Brasil los estudios dan cuenta que este tipo de escuelas se concibe como una política de democratización del acceso a la educación que atiende a estudiantes de distintos niveles etarios (Daros, 2014; Santos, 2017). Sin embargo, el problema que emerge aquí es que la enseñanza se desarrolla en espacios reducidos y con pocos profesionales. Asimismo, se reconocen problemas históricos de la educación multigrado, asociados a escasa infraestructura material, pedagógica, administrativa y de recursos humanos, condiciones de trabajo precarias y formación docente escasa, para desenvolverse en este tipo de establecimientos (Organización de las Naciones Unidas para la Educación, la Ciencia y la Cultura [Unesco], 2004; Daros, 2014; Limera, 2014). Asimismo, los estudios de Santos (2011) y Estrada (2015), profundizan sobre el rol y funciones del profesor en contextos de escuelas multigrado, dando cuenta que: 1) las escuelas multigrado se caracterizan por trabajar con la diversidad social, cultural, étnica, lingüística y etaria propia de sus estudiantes en una misma sala de clases, lo que implica implementar prácticas pedagógicas diversificadas que respondan a sus especificidades particulares (Santos, 2011); 2) la constante actualización curricular y didáctica de los profesores, para responder a las necesidades de los estudiantes (Estrada, 2015); 3) en la práctica pedagógica se identifica la ausencia de orientación para desarrollar la situación de aprendizaje en escuelas rurales multigrado, en la que los profesores enfrentan desafíos y compromisos éticos, sociales y educativos. Esto implica el sacrificio y la creatividad para el docente de manera tal que pueda implementar en su actuar profesional distintas estrategias didácticas, pedagógicas y metodológicas, dando respuesta a las necesidades de la práctica educativa en escuelas multigrados (Oliveira \& Montenegro, 2014; Limeira, 2014). No obstante, esta compleja realidad de escuelas rurales multigrado se constituye a la vez en una oportunidad para la búsqueda de soluciones pedagógicas que emergen de las experiencias de vida cotidiana del docente. Esto abre un espacio para la creación de alternativas, materiales e innovaciones pedagógicas para la superación de las adversidades que surgen en el contexto multigrado (Daros, 2014). En efecto, la realidad y complejidad actual de este tipo de escuela producto de enfrentar mayores desigualdades sociales, culturales y económicas de los estudiantes, afecta negativamente sus resultados académicos, ya que generalmente se ubican en lugares con rasgos de marginación (Herrera \& Buitrago, 2015; Galván \& Espinosa, 2017; Zambrano, 2017).

En el contexto chileno el sistema escolar no se encuentra ajeno a esta realidad educativa, pues existen 3.463 establecimientos educacionales en el sector rural, los que acogen a más de 298.000 estudiantes, que en gran parte de los casos corresponden a escuelas multigrado (Mineduc, 2019). De esta manera, las escuelas multigrado albergan estudiantes de diferentes cursos en una misma sala de clases. Si bien esto puede complejizar la labor docente y los procesos de enseñanza y aprendizaje, muchas veces se convierte en una oportunidad para integrar estrategias de enseñanza innovadoras, contextualizadas y pertinentes al territorio en el que se sitúa la escuela (Mineduc, 2019). Las escuelas multigrado debieran ser capaces de acercar la cultura y el conocimiento a aquellos territorios caracterizados por el aislamiento, precariedad y la influencia de diversos factores que los ubican en situación de desventaja, 
convirtiéndolas así en el inicio del bien común para las comunidades rurales (Cárcamo Vásquez, 2016). Asimismo, debiera ser capaz de ofrecer una educación desde un enfoque educativo intercultural, el cual se basa en el respeto y la valoración de la diversidad de la cultura de origen como configuradores de todas las dimensiones del proceso educativo (Cabrera \& Gallardo, 2013, p. 6). Este enfoque se destaca por el enriquecimiento cultural, teniendo en consideración que en estos contextos conviven principalmente estudiantes indígenas y no indígenas, lo que implica establecer procesos educativos que respeten la diversidad social y cultural presente en el aula (Henríquez, 2013). De esta manera, las políticas de Estado debieran optimizar recursos económicos y humanos para robustecer la educación en escuelas multigrado (Vera, 2015). Y de este modo, revertir las desigualdades entre estudiantes que acceden a la educación rural en relación a aquellos que acceden a la educación en un contexto urbano, en donde estadísticas indican que "Todos los años las escuelas urbanas presentan un puntaje promedio mayor que las escuelas rurales, con una diferencia de 24,8 puntos promedio en 2013 que se ha incrementado a una distancia de 30 puntos en 2015" (Mineduc, 2016, p. 19), lo que estaría influenciado por las prácticas pedagógicas que desarrollan los docentes, así como por la diferencia socioeconómica entre los estudiantes.

\section{Prácticas pedagógicas en contextos de escuelas multigrado}

En el contexto de escuelas multigrados, las prácticas pedagógicas de los docentes constituyen un elemento de vital importancia para desarrollar los procesos de enseñanza y aprendizaje, dadas las características y especificidades propias de este tipo de establecimientos (Vera, 2015). Las prácticas pedagógicas se definen como estrategias y acciones que el profesor realiza para abordar los contenidos curriculares, lo que permite asegurar los aprendizajes de los estudiantes en el marco del sistema educativo escolar chileno. Asimismo, permite que los alumnos logren adquirir las competencias cognitivas, comunicativas y emocionales durante su proceso de escolarización formal. En relación a las competencias cognitivas son entendidas como un saber hacer que todo ser humano adquiere, mediante su proceso de escolarización (Aldaba, 2003). Las competencias comunicativas se refieren al dominio de los principios que rigen el comportamiento del lenguaje y la actuación del estudiante dentro de lo que se espera logre en el sistema escolar (Ronquillo \& Goenaga, 2009). Y, por último, las competencias emocionales debieran permitirle al estudiante un mejor bienestar personal y social, en el marco de su proceso de escolarización formal (Vivas, 2003).

En ese sentido, las prácticas pedagógicas del profesorado deberían permitirle al estudiante realizar un proceso de metacognición en torno a los nuevos conocimientos que adquiere y desarrolla en el marco de la educación escolar. Por otra parte, en el proceso de enseñanza y aprendizaje es importante considerar los factores motivacionales y conductuales. Los factores motivacionales están relacionados con los sentimientos de satisfacción que el individuo presenta a la hora de desarrollar las tareas o actividades en su vida diaria (Amiguinho, 2011). Los factores conductuales, referidos a la forma de actuar de los alumnos dentro del aula, mediados por los profesores, a través de actividades y normas acordes a las características de los educandos; y a los enfoques adaptables al contexto (Abós et al., 2017). De esta manera, las prácticas pedagógicas deben considerar el entorno en que la persona se desenvuelve, es decir, su cultura y modos de vida, lo que permite que los escolares desarrollen un aprendizaje acorde al contexto donde se desenvuelven. A la vez, debiese permitir a los profesores realizar un análisis reflexivo de su propio quehacer profesional.

Las prácticas pedagógicas en el contexto de escuelas multigrado presentan fortalezas y debilidades. Las principales fortalezas se caracterizan por generar un ambiente favorable para el aprendizaje, donde la ayuda entre compañeros y profesores constituye un apoyo en la clase, logrando así un mejor entendimiento del tema que se quiere tratar. A esto se suma que los profesores centran 
el aprendizaje en el alumno, enfatizando la formación en valores, por medio de un aprendizaje participativo, colaborativo y personalizado, desarrollando habilidades claves, con atención a grupos heterogéneos, en el cual se fomenta la autoestima. Igualmente, el docente se presenta como facilitador del aprendizaje por medio de estrategias personalizadas y donde se tiene como miras una mejor relación entre la escuela y la comunidad. Entre las debilidades está el trabajo en condiciones de desventaja económica, dificultando los procesos de enseñanza en los estudiantes. Esto implica la necesidad de crear un ambiente propicio, con las condiciones básicas para desarrollar habilidades, actitudes y situaciones didácticas que faciliten al alumnado construir aprendizajes significativos (Romero \& Vásquez, 2004; Boix, 2011; Enayati et al., 2016). Asimismo, existen precarias condiciones materiales de trabajo y una escasa orientación pedagógica y metodológica para el trabajo en este tipo de aulas, lo que genera que los profesores enfrenten serias limitaciones para conducir los procesos de aprendizaje (Rodríguez, 2004). De este modo, las escuelas son pensadas para responder a la necesidad de la población vulnerable; sin embargo, existe una escasa formación inicial docente para enseñar en un aula multigrado y se carece, además, de recursos pedagógicos adecuados al contexto social y cultural donde se desarrollan los procesos de enseñanza y aprendizaje (Henríquez, 2013; Vera, 2015; Díaz et al., 2016).

\section{Metodología}

La investigación es de carácter cualitativo, que tiene a la base la necesidad de conocer y comprender la realidad de los sujetos, según sus propios significados (Flick, 2012). Se sustenta en un paradigma interpretativo que permite comprender la realidad como un hecho dinámico e interactivo en el marco de las particularidades de un contexto específico, según las interpretaciones y construcción de significados desde los propios sujetos que allí participan (Rodríguez et al., 1996; Ricoy, 2006). El contexto de estudio es una escuela rural multigrado ubicada en una comunidad mapuche de La Araucanía de dependencia municipal. Esta escuela ofrece Educación Parvularia y Básica, de primero a sexto año básico. La escuela consta con una matrícula de alrededor de 40 estudiantes. Los participantes fueron 3 profesores de la escuela, un hombre y dos mujeres, los que coinciden con los criterios de inclusión. Entre estos, desempeñarse actualmente en el establecimiento y ser profesores de Educación Básica. La edad de los participantes fluctúa entre los 35 y 45 años. Cuentan con una experiencia entre uno y tres años de prácticas pedagógicas en el establecimiento.

El instrumento para la recolección de datos e información es la entrevista semiestructurada que consultó sobre los desafíos socioeducativos que enfrentan los profesores en sus prácticas pedagógicas en escuelas multigrados. La entrevista tuvo una duración aproximada de 30 minutos, previa firma de los consentimientos informados, para resguardar los aspectos éticos (Mondragón, 2009). La técnica y procedimiento de análisis de la información utilizado es la teoría fundamentada, a través de un proceso de codificación abierta y axial (Mondragón, 2009). La codificación abierta permitió el resultado de una lista de códigos y categorías que se asignaron al texto, de acuerdo a las unidades de significado atribuidas por los participantes. La codificación axial permitió relacionar y generar una categoría central respecto de los temas principales que emergieron en lo que concierne al objeto de estudio. Esto permitió identificar la categoría central, subcategorías y códigos que permiten comprender los principales desafíos que enfrentan en sus prácticas pedagógicas los profesores que se desempeñan en escuelas multigrado.

\section{Resultados}

La presentación de los resultados se organiza considerando como categoría central las experiencias pedagógicas en aulas multigrado, y sus respectivas subcategorías que emergen con mayor recurrencia en el testimonio de los profesores, siendo éstas las siguientes: 1) Desafíos educativos; 2) Desafíos sociales; y 3) Prácticas pedagógicas (Tabla 1). 


\begin{tabular}{ccc}
\hline Subcategoría & Recurrencia & Porcentaje \\
Desafíos educativos & 17 & $28 \%$ \\
Desafíos sociales & 13 & $22 \%$ \\
Prácticas pedagógicas & 30 & $50 \%$ \\
\hline Total & 60 & $100 \%$ \\
\hline
\end{tabular}

Fuente: Elaboración propia.

La categoría central Experiencias pedagógicas en aula multigrado y sus códigos se presentan en la siguiente figura (Figura 1).

\section{FIGURA 1}

\section{EXPERIENCIAS PEDAGÓGICAS EN AULA MULTIGRADO}

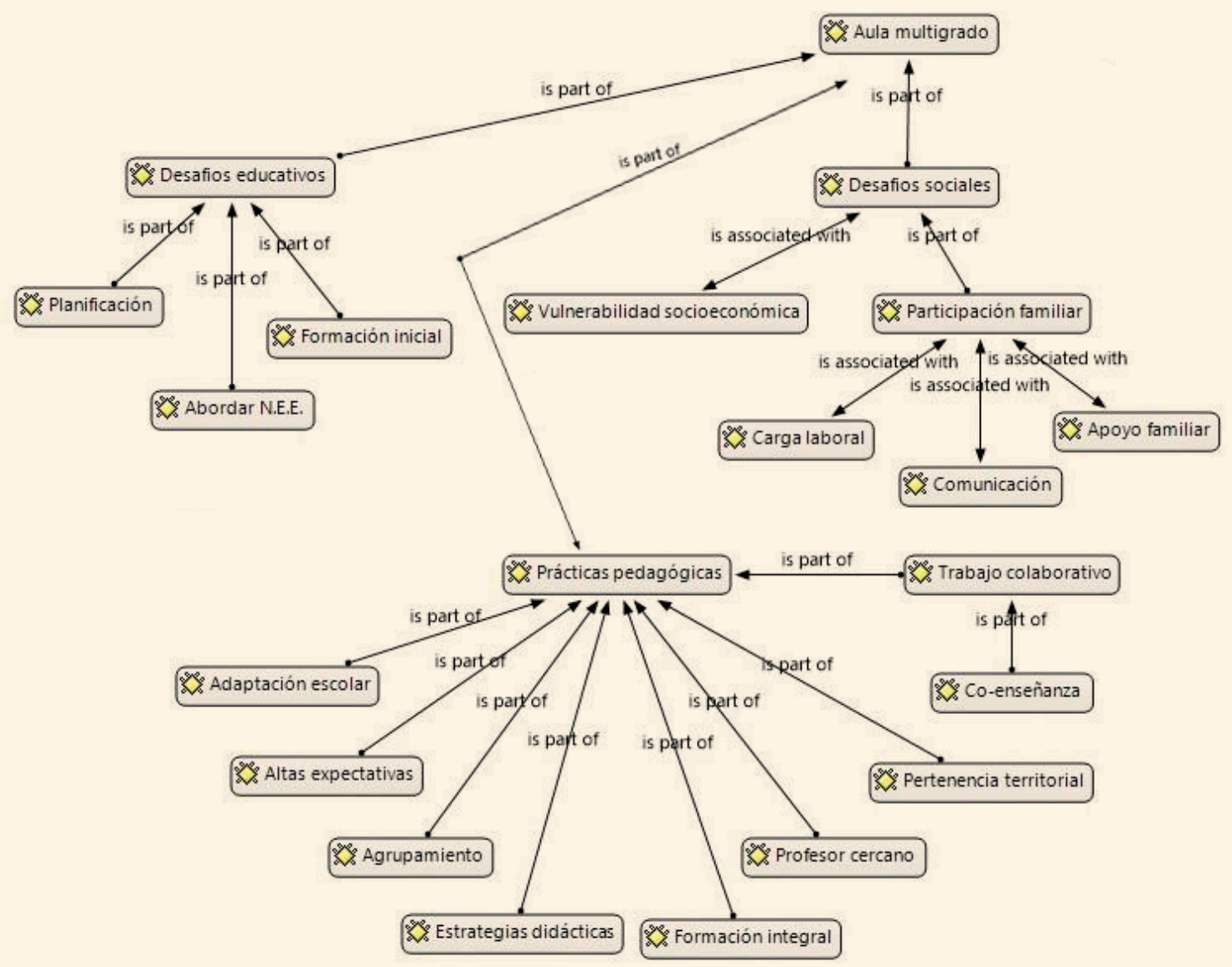

Fuente: Elaboración propia con apoyo del software Atlas ti.7.2.

\section{Desafíos educativos}

La subcategoría desafíos educativos se relaciona con las principales dificultades que evidencian los profesores al momento de enseñar en un aula multigrado. Dicha subcategoría obtiene una frecuencia de $28 \%$ de un total de 60 recurrencias. La subcategoría se compone de los siguientes códigos. El primero es el código planificación del proceso de enseñanza y aprendizaje, que refiere a las formas que asume el 
profesor para organizar los contenidos educativos curriculares, los objetivos de aprendizaje y desarrollo de competencias y habilidades exigidas por el currículo escolar, para apoyar a los estudiantes en el éxito escolar. Respecto al código planificación, un testimonio plantea que "Lo más difícil de la educación en escuelas multigrado es cuando llegas al momento de planificar, porque tienes que reunir todos los objetivos, buscar los que se parezcan, conectarlos y trabajarlos todos juntos en el aula" (P1EB [1:438-1:834]). El relato de la profesora da cuenta del desafío que implica la planificación dentro del contexto de aula multigrado, pues requiere de la selección e integración de objetivos de distintos niveles educativos para dar respuesta a las necesidades de los estudiantes, lo que implica una articulación entre niveles escolares. Asimismo, en ocasiones se carece del dominio de estrategias y conocimiento disciplinar para llevar a cabo este procedimiento durante la enseñanza.

El segundo código refiere a cómo el profesor aborda las Necesidades Educativas Especiales (N.E.E), utilizando diferentes formas y estrategias, didácticas, pedagógicas y metodológicas, para asegurar a los estudiantes progresar en su proceso de aprendizaje. Dentro de las N.E.E. más recurrentes en la escuela, se encuentran el Funcionamiento Intelectual Limítrofe (F.I.L.), el Trastorno Específico del Lenguaje (T.E.L.), el Trastorno del Espectro Autista (T.E.A.) y Trastornos por Déficit de Atención e Hiperactividad (T.D.A.H.). Dentro del discurso de los profesores, abordar las N.E.E. es un reto, ya que implica movilizar diferentes recursos, como el uso de material concreto y estrategias referidas a acciones del profesor, o la creación de espacios propicios para el aprendizaje. Al respecto, un profesor menciona que: "[lo más difícil en aula multigrado es] ... llevar el trabajo de los niños con necesidades educativas especiales, porque el curso mío es el que tiene más N.E.E. y no tengo las herramientas personales para poder abordarlas" (P2EB [1:2450-1:2564]). De acuerdo al testimonio del profesor, podemos inferir que este hecho se relaciona con la formación inicial que tuvieron los docentes de Educación General Básica, quienes reconocen carecer de herramientas y conocimientos disciplinarios, didácticos y metodológicos para atender las necesidades educativas especiales de los estudiantes con los que trabajan. Esto los limita y les coarta la posibilidad de generar procesos de enseñanza que favorezcan la inclusión educativa y la participación plena de los estudiantes que integran su aula. Frente a dicho desafío, los profesores expresan en sus testimonios que se han buscado alternativas, mediante la implementación del Programa de Integración Escolar. En relación a esto, una profesora señala: "Ella [Educadora Diferencial] sabe bien, . . . los problemas que tienen los chiquillos, sus necesidades educativas especiales, entonces ella sabe con qué trabajar, qué trabajar o por qué tenemos que trabajar con esto" (P2EB [9:1728-9:2007]). De acuerdo al relato, la profesora de Educación Diferencial cuenta con las estrategias y conocimientos para abordar las N.E.E. que presentan los estudiantes, ya que ella es quien posee la formación profesional para ello. Asimismo, evidenciamos que existe una delegación de responsabilidades en la que la profesora asume que es sólo la educadora diferencial quien debe hacerse cargo de estas actividades. Lo que es preocupante, si consideramos que dentro de los lineamientos del Programa de Integración Escolar se asume que el trabajo en aula debiese sustentarse en una co-enseñanza entre las profesionales para atender a la diversidad existente en el aula.

En relación al código de Formación Inicial Docente, éste se refiere a la forma en que los futuros profesores están formados dentro de las universidades, siendo fundamental en este proceso la adquisición de competencias y contenidos teóricos para poder desarrollar prácticas pedagógicas que respondan a las necesidades educativas de los estudiantes y a las realidades del contexto educativo actual. Es por ello que, en esta instancia de formación, es cuando el profesor debe adquirir las herramientas necesarias para desarrollar una labor transformadora y entregar una enseñanza de calidad (Gaete et al., 2016). En torno a la formación inicial una profesora menciona que: "La Universidad no nos prepara, por lo menos a mí no me preparó para trabajar en aula multigrado, yo salí de la Universidad y cuesta trabajar en un aula multigrado y poder planificar adecuadamente" (P1EB [4:598-4:801]). Podemos inferir que, si bien la universidad brinda ciertas herramientas, éstas no responden efectivamente a la realidad 
que se encuentra dentro del contexto laboral de los profesores. Asimismo, es necesario que las instituciones de Educación Superior consideren las experiencias de los profesores en ejercicio para desarrollar mallas curriculares efectivas y actualizadas, acordes a la realidad social, cultural y educativa que enfrentan en su quehacer profesional.

\section{Desafíos sociales}

La subcategoría desafíos sociales se relaciona con las principales dificultades externas que enfrentan los profesores en el contexto educativo, las que influyen en el proceso de enseñanza y aprendizaje de los alumnos y afectan su rendimiento. Dicha subcategoría obtiene una frecuencia de $22 \%$ de un total de 60 recurrencias. La subcategoría se compone de los siguientes códigos. El primer código, vulnerabilidad socioeconómica, refiere a un bajo nivel de ingresos económicos a nivel familiar, que trae consigo dificultades que les impiden su pleno bienestar; por ejemplo, la dificultad de acceder a atención de salud y educación de calidad (Gil-Lacruz et al., 2015). De acuerdo al relato de los profesores los estudiantes se encuentran el mayor tiempo solos debido a que sus padres están fuera del hogar por motivos de trabajo. $\mathrm{Al}$ respecto un profesor menciona: "Tenemos niños que son del campo y otros de acá del sector de Chivilcan, entonces son niños demasiado vulnerables, que prácticamente se crían solos" (P3EB [3:453-3:598]). En torno al relato, la vulnerabilidad de las familias es un tema complejo, puesto que el lugar de residencia de los estudiantes es caracterizado por contar con pocos recursos económicos, lo que obliga a los padres a buscar alternativas laborales en otros sectores para responder a las necesidades básicas de sus hijos y familias. Esto genera que los niños se encuentren solos la mayor parte del tiempo, pues sus padres se enfocan en buscar los recursos económicos necesarios, dejando en cierta forma de lado el papel educativo que cumplen en el proceso de aprendizaje de sus hijos.

En relación al segundo código, de la participación familiar, refiere a la relación e implicación de las familias en las escuelas, lo que contribuye a la mejora de los procesos y resultados educativos (Calvo et al., 2016). En su discurso una profesora señala: "Nosotros tratamos lo máximo de involucrar a la familia, pero hay poco apoyo, la mayoría de los apoderados trabajan, mamá y papá, algunos chicos que viven solo con los abuelos; entonces hay poco apoyo" (P1EB [1:1599-1:1800]). De acuerdo al relato, existe una baja participación familiar, la cual está relacionada principalmente a las condiciones laborales de los padres, puesto que se ven enfrentados a largas jornadas laborales. Situaciones que limitan su implicación en el proceso de aprendizaje, puesto que deben priorizar otras necesidades por sobre el aprendizaje de sus hijos. Otro testimonio refuerza la cita anterior, señalando que: "Tratamos de comunicarnos a través de cuadernos, reuniones, citaciones para conversar y de esa manera tratamos de involucrarlos un poco, porque la gran mayoría tiene tanto trabajo que se desliga un poco del proceso" (P1EB [1:1872-1:2077]). El relato da cuenta del esfuerzo por parte de los profesores para involucrar a las familias, lo que no siempre se logra. Esto se podría explicar por los canales de comunicación utilizados, que suelen ser formales, que no dan paso a una comunicación fluida, considerando las características de las familias, entre ellas la vulnerabilidad socioeconómica y la carga laboral. De igual forma, influye el apoyo de los padres, lo que se evidencia cuando un profesor señala: "Es un tema en el hogar, pues, yo creo que se está hablando menos o no se conversan algunas cosas y me he dado cuenta que esto está pasando cada vez más" (P3EB [1:957-1:1150]). Esto se podría explicar hipotéticamente por una mala comprensión de apoyo por parte de los padres en el proceso, donde ellos participan en reuniones de padres y apoderados, utilizando esa instancia para conversar del proceso educativo con los profesores. 


\section{Prácticas pedagógicas}

La subcategoría de prácticas pedagógicas se relaciona con las estrategias y acciones que desarrollan los profesores para guiar el proceso de enseñanza y aprendizaje en un aula multigrado con el fin de desarrollar en los estudiantes competencias cognitivas, emocionales y sociales. Dicha subcategoría obtiene en el testimonio de los profesores una frecuencia de 50\% de un total de 60 recurrencias. La subcategoría se asocia a los siguientes códigos. El primer código es el de adaptación escolar, que hace referencia al proceso adaptativo en el cual el niño pasa de una unidad de convivencia más conocida como la escuela de origen, a otro ámbito desconocido y nuevo, que suele ser otro establecimiento educativo. Al respecto, una profesora menciona que: "Tratas de que ellos se lleven una buena formación del establecimiento y a la vez, que no sea el cambio tan brusco al llegar a otro colegio, porque aquí se van de sexto, igual se van chiquititos" (P2EB [2:2864-3:141]). Es decir, ellos lo que desean de sus estudiantes es generar una igualdad de aprendizaje, con la posibilidad de que al cambiar de institución logren adaptarse de manera efectiva.

El segundo código se refiere a altas expectativas, éste hace referencia al comportamiento positivo que transmite el profesor a sus estudiantes, tanto verbal como no-verbal. Esto genera sentimientos de autoconfianza en los estudiantes y mayor satisfacción, compromiso y dedicación en tareas académicas por parte del profesor. Respecto a esto una profesora señala: "Yo quiero lo mejor, para primero básico, quiero que todos manejen la numeración, que sepan leer a fin de año" (P2EB [1:1192-1:1374]). Esto refleja el compromiso de la profesora ante las necesidades educativas de los estudiantes, pues, al tener altas expectativas, desarrollará acciones que fomenten la participación de cada uno, potenciando su autosuficiencia, confianza y colaboración, reconociendo los intereses y gustos para vincular los contenidos disciplinarios. Ante lo anterior, una profesora menciona: "Yo los quiero ver en la universidad, con alguna profesión, que salgan del país y así muchas otras cosas más" (P1EB [1:1387-1:1499]). Es así que los profesores contribuyen en generar aprendizajes de calidad, entregando herramientas educativas para que los estudiantes consigan mejores oportunidades en el transcurso de sus vidas.

El tercer código, agrupamiento, refiere a la distribución que se realiza en el aula, utilizando una mejor estructuración de los materiales, una mayor organización y entorno físico que entrega oportunidades y posibilidades de trabajo a los estudiantes (Bermejo Cepeda et al., 2010). Una profesora, al referirse a agrupamiento, señala que distribuye a los estudiantes: "En filas a veces trabajamos en forma grupal, a veces por estaciones, vamos variando según la actividad" (P2EB [(3:1894-3:2012]). De acuerdo al relato, la profesora organiza a los estudiantes de manera que todos puedan adquirir aprendizaje y participar durante las clases, ya sea organizando el entorno, ordenándolos en filas o ajustándose a la situación que lo amerita. A su vez, permite generar relaciones positivas, pues los estudiantes interactúan unos con otros, tienden a ser más empáticos, aprenden a conocerse y así tener más capacidades para resolver situaciones.

El cuarto código de recursos didácticos se entiende como el apoyo pedagógico que refuerza la labor del profesor, optimizando el proceso de enseñanza y aprendizaje. Estos recursos pueden ser materiales tanto físicos como virtuales, que deben despertar el interés de los estudiantes y adecuarse a sus características físicas y psíquicas. Dentro de su discurso una profesora señala que: "trabajamos con muchos recursos materiales en el colegio, ... con textos, con recursos visuales, con recursos que se puedan manipular" (P1EB [3:764-3:962]). Desde el relato, es posible inferir que la profesora diversifica la enseñanza, empleando variadas estrategias y recursos didácticos, con el fin de que todos sus estudiantes aprendan el contenido. Reconociendo que cada estudiante tiene distintas formas y ritmos de aprendizaje, por lo que es necesario brindar una enseñanza diversificada, que dé respuesta a la heterogeneidad característica de un aula multigrado. 
El quinto código, de formación integral, se refiere al proceso mediante el cual se logra que los seres humanos, en colaboración con otros, desarrollen todo su potencial, con el fin de optimizar las oportunidades ofrecidas por el mundo, la cultura y sociedad. La formación integral se relaciona con la modalidad en que los profesores forman a los estudiantes, concientizando en valores para actuar en la sociedad y a su vez les permita desenvolverse en diversos contextos. Respecto a esto un profesor señala: "Se trata de hacer una persona integral, que pueda trabajar tanto en el campo, como en la ciudad" (P3EB [3:981-3:1086]). Donde la labor docente genera herramientas pertinentes a la realidad contextual y cultural de sus estudiantes.

Con respecto al sexto código, el del profesor cercano, refiere a la relación educativa que establece el docente con sus estudiantes con base en la estimulación de los educandos, para comprender el mundo que los rodea, aprendiendo a vivir con dignidad, desarrollando sus capacidades profesionales y comunicándose con los demás (De Cleves et al., 2004). En relación a este código un profesor señala que: “. . . es importante la cercanía en realidad con ellos, no ser tan dura tampoco, si son niños que les faltan demasiado cariño, entonces para qué ser una persona tan lejana, si ellos necesitan igual cariño (P3EB [6:1036-6:1242]). Al establecer una relación educativa cercana con los estudiantes se genera una mejor convivencia y clima propicio para el aprendizaje entre el profesor y los estudiantes. Esto facilita el bienestar de cada uno y, además, permite involucrar a los padres en el proceso escolar de sus hijos.

En relación al séptimo código, pertenencia territorial, éste hace referencia a la promoción y valoración que desarrollan los profesores en sus estudiantes, al sentido de pertenencia del individuo al territorio y del proceso de formación de identidad que experimenta al sentirse parte de dicho territorio (Romero \& Vásquez, 2004). Con respecto a esto, un profesor señala: "Eso estamos tratando de hacer con los niños, que se queden donde viven y trabajan y se desarrollen y formen su familia y todo eso" (P3EB [3:1377-3:1506]). Por tanto, el profesor quiere lograr que sus estudiantes se sientan parte de su territorio, logrando que estudien en él y que en un futuro trabajen en el mismo solventando su propia economía. En relación a lo anterior una profesora agrega "Respetando las culturas de los chicos, hablamos de la cultura de origen, tratando de respetar y que entre ellos también se respeten, de donde provenga cada uno, el bagaje que traiga cada uno todo desde el respeto" (P3EB [3:97-3:625]). Con respecto a este testimonio la pertenencia territorial cumple un rol fundamental en los estudiantes que se educan en este tipo de establecimiento, ya que aprenden mucho más de su cultura, se genera un ambiente más cercano y de respeto entre los estudiantes y fundamentalmente se quedan en su territorio.

Finalmente, el código del trabajo colaborativo se entiende como un proceso en el que un individuo aprende más de lo que aprendería por sí solo, fruto de la interacción de los integrantes de un equipo, quienes saben diferenciar y contrastar sus puntos de vista. De tal manera que llegan a generar un proceso de construcción de conocimiento co-construido, tanto con sus pares como con sus profesores (Revelo-Sánchez et al., 2018). Al respecto en un testimonio se señala que: "Trabajamos todo en conjunto, vamos analizando las necesidades y en conjunto buscamos estrategias para abordar a todos" (P1EB [1:976-1:1096]). El trabajo colaborativo se destaca como un facilitador dentro del establecimiento, constituyéndose en una herramienta para desarrollar una labor pedagógica más efectiva que responde a las necesidades de una escuela multigrado. Al respecto una profesora menciona que "Dentro del aula, hacemos clases colaborativas, con la Educadora Diferencial trabajamos en conjunto, y de esa manera buscamos las mejores estrategias para los niños que tengan necesidades educativas y para el curso en general" (P1EB [4:1969-4:2262]). En relación al testimonio se deduce que existe un proceso de co-enseñanza dentro del aula; ésta es una de las estrategias empleadas para poder responder a las necesidades educativas de cada estudiante. 


\section{Discusión y conclusión}

Los resultados del estudio nos permiten constatar que los profesores de Educación Básica que desarrollan sus prácticas en un aula multigrado se ven enfrentados en su quehacer pedagógico a desafíos socioeducativos. Tales como la vulnerabilidad socioeconómica que, según Díaz et al. (2016) se expresa en las carencias sociales y económicas que poseen las familias asociadas con el contexto de pobreza, donde el compromiso familiar no tiene relación con la práctica educativa desarrollada por el docente. Más bien se relaciona con el grado en que la familia anima el aprendizaje para salir de la pobreza. Dicha realidad se evidencia en la escuela investigada, donde las familias en su gran mayoría son vulnerables, y esta condición limita su participación en las actividades educativas de sus hijos, influyendo en el proceso de enseñanza y aprendizaje, pues existe baja interacción entre la familia-escuela. Otro de los desafíos, es una Formación Inicial Docente que no entrega las herramientas necesarias para trabajar en un aula multigrado, lo que limita el desarrollo de prácticas pedagógicas que respondan a las especificidades propias del territorio en el que se sitúa la escuela. Asimismo, los contenidos académicos proporcionados por las instituciones universitarias suelen ser descontextualizados, pues no logran responder a las demandas de este medio. Esto es coherente con lo planteado por Arias-Ortega et al. (2018), quienes plantean que la Formación Inicial Docente en Chile suele ser monocultural, careciendo de saberes y conocimientos locales, propios de las culturas de cada territorio. A lo que se le suma el desconocimiento pedagógico, didáctico y metodológico para abordar las necesidades educativas especiales que pudiesen emerger en la sala de clases.

En el marco de los desafíos socioeducativos de las prácticas pedagógicas, los resultados de investigación nos permiten constatar que los profesores intentan abordar por medio del trabajo colaborativo y la co-enseñanza los procesos de enseñanza y aprendizaje, para fortalecer sus procesos de planificación en articulación con la profesora de Educación Diferencial. En concordancia con lo anterior, la co-enseñanza constituye una estrategia que favorece el aumento de la eficacia de las escuelas, ya que los profesores en este contexto se deben coordinar, compartir, mostrar y distribuir las funciones (Agencia de Calidad de la Educación, 2018). Esto ha favorecido el cumplimiento de la planificación curricular, producto de las competencias, tales como la flexibilidad, autonomía, respeto a la diversidad y trabajo colaborativo, que desarrollan los profesores en el aula multigrado.

En este mismo sentido, las prácticas pedagógicas de los profesores están marcadas por las interacciones que se establecen dentro de la sala de clases con los estudiantes, donde el profesor tiene un rol de mediador en el proceso de enseñanza y aprendizaje (Morillo \& Quijano, 2016). En torno a las acciones y percepciones más recurrentes de los profesores de la escuela multigrado, se constata que cuentan con altas expectativas en el desempeño académico que pueden lograr los estudiantes, propiciando procesos educativos de calidad en torno a la adquisición de aprendizajes significativos. Dicha perspectiva se relaciona con lo planteado por Chile (Mineduc, 2008) en el Marco para la Buena Enseñanza (MBE), donde se menciona que es fundamental que el profesor al interior de las salas de clases destaque las fortalezas de sus estudiantes, considerando y valorizando sus características e intereses, lo que se relaciona con la disposición a comprometerse con los contenidos que enseña y con lograr que sus estudiantes se motiven y valoren lo aprendido.

En consecuencia, los resultados de investigación nos permiten sostener que la escuela multigrado en contexto rural es un ambiente propicio de aprendizaje, y que se maximizarían sus resultados si se le brindaran las posibilidades de mejora, asociadas a: 1) adecuada infraestructura; 2) apoyo a la formación continua de los profesores, aportando con entrega de herramientas didácticas, pedagógicas y metodológicas para transformar sus prácticas educativas; 3 ) entrega de recursos didácticos pertinentes y contextualizados a la realidad de un aula multigrado; 4) dejar de lados las estigmatizaciones e invisibilización por parte del Estado de la compleja realidad que viven las escuelas ubicadas en sectores 
rurales, que generalmente se encuentran ubicadas en comunidades indígenas. Esta invisibilización de las problemáticas educativas en contextos rurales contribuyen a la perpetuación de la pobreza y desigualdad educativa entre indígenas y no indígenas. Por lo que es necesario cuestionar el status quo de la escuela para erradicar estas prácticas; y 5) priorizar el planteamiento de políticas públicas que respondan adecuadamente a las necesidades que presenta la educación rural, lo que permita igualar las oportunidades entre los estudiantes sin importar su nivel social o ubicación geográfica. Este conjunto de acciones puede contribuir para una mejor toma de decisiones y para mejorar la calidad de la educación en contexto rural, la que se ha caracterizado históricamente por su diversidad social y cultural.

\section{Agradecimientos}

Agradecemos al Proyecto PROFONDECYT 2019 PF-KA-05, financiado por la Vicerrectoría de Investigación y Posgrado de la Universidad Católica de Temuco, y al proyecto FONDECYT DE INICIACIÓN N ${ }^{\circ}$ 11200306, financiado por la Agencia Nacional de Investigación y Desarrollo de Chile (ANID).

\section{Referencias}

Abós, P., Fuguet, J., \& Torres, C. (2017). Aprendizaje y escuela rural: La visión del alumnado. Revista Electrónica de Educación, 1(49), 1-17. http://www.scielo.org.mx/scielo.php?script=sci_arttext\&pid=S1665109X2017000200006\&lng=es\&nrm=iso

Agencia de Calidad de la Educación. (2018). Informe nacional de calidad de la educación 2018. Agencia de la Calidad de la educación. http://archivos.agenciaeducacion.cl/informe_nacional_de_la_calidad_de_ la_educacion_2018.pdf

Aldaba, A. (2003). Las competencias cognitivas y el perfil del aprendiz exitoso. Investigación Educativa Duranguense, 1(2), 11-22. https://dialnet.unirioja.es/servlet/articulo?codigo=3683582

Ames, P. (2004). Las escuelas multigrado en el contexto educativo actual: Desafíos y posibilidades. GTZProeduca, 2(1), 5-47. http://www.grade.org.pe/creer/archivos/Ames-Patricia.-2004-Las-escuelasmultigrado-en-el-contexto-educativo-actual-1.pdf

Amiguinho, A. (2011). La escuela en el medio rural: Educación y desarrollo rural. Revista de Curriculum y Formación del Profesorado, 15(2), 25-37. https://dialnet.unirioja.es/servlet/articulo?codigo $=4089454$

Arias-Ortega, K. (2019). Relación educativa entre el profesor mentor y el educador tradicional en la educación intercultural [Tesis Doctoral]. Universidad Católica de Temuco.

Arias-Ortega, K., \& Quintriqueo-Millán, S. (2020). Higher education in mapuche context: The case of La Araucanía, Chile. Revista Electrónica Educare, 24(2), 1-19. https://doi.org/10.15359/ree.24-2.1

Arias-Ortega, K., Quintriqueo, S., \& Valdebenito, V. (2018). Monoculturalidad en las prácticas pedagógicas en la formación inicial docente en La Araucanía, Chile. Educação e Pesquisa, 44(1), 1-19. https://doi. org $/ 10.1590 / \mathrm{s} 1678-4634201711164545$

Arteaga Martinez, P., Williamson Castro, G., Berrouet Marimón, F. R., \& Segundo Cabello, A. (2017). La concentración de escuelas rurales en América Latina. In Anais do 14 Congreso Nacional de Investigación Educativa, 1(1), 1-36. http://www.comie.org.mx/congreso/memoriaelectronica/v14/ doc/simposios/1399.pdf

Bermejo Cepeda, A., Gómez Lara, E., Ocaña Serrano, A., Sánchez González, R., Sebastián Heredero, E. (2010). Análisis de la organización del aula en distintos niveles educativos. Revista Ibero-Americana de Estudos em Educação, 5(2), 135-153. https://www.sumarios.org/artigo/an\%C3\%A1lisis-de-laorganizaci\%C3\%B3n-del-aula-en-distintos-niveles-educativos-estudio-de-casos

Boix, R. (2011). ¿Qué queda de la escuela rural? Algunas reflexiones sobre la realidad pedagógica del aula multigrado profesorado. Revista de Currículum y Formación del Profesorado, 2(15), 13-23. http://www. ugr.es/ recfpro/rev152ART1.pdf 
Bustos, A. (2014). La didáctica multigrado y las aulas rurales: Perspectivas y datos para su análisis. Revista Innovación Educativa, 1(24), 119-131. https://dialnet.unirioja.es/servlet/articulo?codigo=4920455

Cabrera, I., \& Gallardo, T. de J. (2013). Educación intercultural del estudiante universitario: El enfoque de formación humanístico intercultural. Revista Electrónica Actualidades Investigativas en Educación, 13(3), 1-34. https://www.redalyc.org/pdf/447/44729878002.pdf

Calvo, M., Verdugo, M., \& Amor, A. (2016). La participación familiar es un requisito imprescindible para una escuela inclusiva. Revista Latinoamericana de Educación Inclusiva, 10(1), 99-113. https://scielo. conicyt.cl/pdf/rlei/v10n1/art06.pdf

Cárcamo Vásquez, H. (2016). Microcentros de escuelas rurales de la provincia de Nuble, Chile: Representaciones que posee el profesorado respecto de su impacto en el quehacer pedagógico en el escenario de la nueva ruralidad. Sinéctica, (47) http://www.scielo.org.mx/scielo.php?script=sci_arttext\&pid=S1665-109X2 016000200010\&lng=es\&tlng=es

Castillo-Miranda, S., Williamson, G., \& Hidalgo-Standen, C. (2017). La evaluación del desempeño docente desde la perspectiva de profesores de educación rural. Educación y Educadores, 20(3), 364-381. http:// www.scielo.org.co/pdf/eded/v20n3/0123-1294-eded-20-03-00419.pdf

Chávez, L., \& García, J. (2013). Las escuelas unidocentes en Costa Rica: Fortalezas y limitaciones. Revista Educación, 37(1), 1-27. https://www.redalyc.org/pdf/440/44028564001.pdf

Daros, C. da M. (2014). Escolas multisseriadas: A experiência internacional e reflexões para o caso brasileiro. Ensaio: Avaliação e Políticas Públicas em Educação, 22(82), 57-88. https://www.scielo.br/pdf/ensaio/ v22n82/a04v22n82.pdf

De Cleves, N., Velásquez, B., \& Calle, M. (2004). El maestro como formador y cultor de la vida. Revista de Humanidades Tabula Rasa, 1(2), 263-281. https://www.redalyc.org/pdf/396/39600215.pdf

Díaz, R., Muñoz, S., \& Osses, S. (2016). Factores e interacciones del proceso de enseñanza-aprendizaje en contextos rurales de la Araucanía, Chile. Estudios Pedagógicos, 42(3), 111-128. https://scielo.conicyt. $\mathrm{cl} / \mathrm{pdf} / \mathrm{estped} / \mathrm{v} 42 \mathrm{n} 3 /$ art06.pdf

Enayati, T., Zameni, F., \& Movahedian, M. (2016). Classroom management strategies of multigrade schools with emphasis on the role of technology. Interdisciplinary Journal of Virtual Learning in Medical Sciences, 7(2), 1-10. https://doi.org/10.5812/ijvlms.12161

Estrada, M. (2015). Multigrado en derecho propio. Revista Latinoamericana de Estudios Educativos, 45(2), 43-62. https://www.redalyc.org/pdf/270/27039624003.pdf

Flick, U. (2012). Introducción a la investigación cualitativa. Morata.

Gaete, A., Gómez, V., \& Bascopé, M. (2016). ¿Quéle piden los profesores a la formación inicial docente en Chile? Temas de la Agenda Pública, 1(86), 1-17. https://politicaspublicas.uc.cl/wp-content/uploads/2016/06/ Qu\%C3\%A9-le-piden-los-profesores-a-la-formaci\%C3\%B3n-inicial-docente-en-Chile.pdf

Gallardo, M. (2011). La escuela de contexto rural: ¿De la diferencia a la desigualdad? Revista Iberoamericana de Educación, 55(5), 1-10. https://doi.org/10.35362/rie5551566

Galván, L., \& Espinosa, L. (2017). Diversidad y prioridades educativas en escuelas multigrado: Estudio de caso en México. Revista Electrónica de Educación, 1(49), 1-19. http://www.scielo.org.mx/scielo. php?script $=$ sci_arttext\&pid=S1665-109X2017000200005\&lng=es\&nrm=iso

Gil-Lacruz, M., Gil-Lacruz, A., \& Aguilar, I. (2015). Vulnerabilidad socioeconómica en un vecindario como determinante de la utilización y frecuentación de servicios sanitarios. Enfermería Global, 14(38), 178-189. DOI: https://doi.org/10.6018/eglobal.14.2.201951

Henríquez, L. (2013). Cinco décadas de transformaciones en la Araucanía Rural. Revista Latinoamericana, $12(34), 147-164$.

Herrera, L., \& Buitrago, R. (2015). Educación rural en Boyacá, fortalezas y debilidades desde la perspectiva del profesorado. Praxis \& Saber, 6(12), 169-190. https://doi.org/10.19053/22160159.3768

Juárez, D. (2012). Educación rural en Finlandia: experiencias para México. Revista de Investigación Educativa, 1(15), 140-154. https://www.redalyc.org/pdf/2831/283123579008.pdf 
Ley n. 20370, de 12 de septiembre de 2009. (2009). Establece la ley general de educación. Mineduc, 2009. https://www.ayudamineduc.cl/ficha/lge-ley-general-de-educacion-4

Limeira, K. (2014). Educação rural no Brasil: Um olhar a partir do contexto histórico. In Anais, do 4 Congresso Sergipano de História \& Encontro Estadual de História da ANPUH/SE: O cinquentenário do golpe de 64. Instituto Histórico e Geográfico de Sergipe, Aracaju. http://www.encontro2014.se.anpuh.org/ resources/anais/37/1424131440_ARQUIVO_KatiaMariaLimeiraSantos.pdf

Luria, A., Leontiev, A., \& Vigotsky, L. (2004). Psicología y pedagogía. Akal.

Ministerio de Educación. (2008). Marco para la buena enseñanza. Mineduc. https://www.docentemas.cl/docs/ MBE2008.pdf

Ministerio de Educación. (2016). Matemática - 4 Básico. Mineduc. http://oes.ufro.cl/index.php/oes-ufro/ estadisticas/sociales/datos-historicos/download/10-datos-historicos/372-resultados-simce-cienciassociales-y-ciencias-naturales-8-basico-en-escuelas-rurales-de-la-araucania

Mondragón, L. (2009). Consentimiento informado: Una praxis dialógica para la investigación. Revista de Investigación Clínica, 61(1), 73-82. https://www.ncbi.nlm.nih.gov/pmc/articles/PMC2788237/

Morillo, O., \& Quijano, O. (2016). Concepciones y prácticas docentes sobre la diversidad como base para la innovación pedagógica: Un estudio de caso en el colegio Vasco Núñez de Balboa. Revista Plumilla Educativa, 18(2), 318-336. https://dialnet.unirioja.es/servlet/articulo?codigo=5920218

Niemi, H. (2015). Desarrollo profesional docente en Finlandia: Hacia un enfoque más holístico. Psicología, Sociedad y Educación, 7(3), 387-404. https://dialnet.unirioja.es/servlet/articulo?codigo=6360144

Oliveira, M. B. de, \& Montenegro, J. (2014). A educação do campo no contexto do modelo de desenvolvimento rural no Brasil: O princípio educativo do trabalho como alternativa. Revista Pegada, 15(1), 171-207. https://revista.fct.unesp.br/index.php/pegada/article/viewFile/2671/2628

Organização das Nações Unidas para a Educação, a Ciência e a Cultura - Unesco. (2004). Educación para la población rural en Brasil, Chile, Colombia, Honduras, México, Paraguay y Perú. Proyecto FAOUNESCO-DGCS/ITALIA-CIDE-REDUC. http://www.fao.org/3/y5517s/y5517s00.pdf

Pérez, M., García, I., \& Quijano, R. (2018). Una aproximación a la realidad de los centros públicos rurales en Andalucía. Tendencias Pedagógicas, 32(1), 147-160. https://revistas.uam.es/tendenciaspedagogicas/ article/view/tp2018.32.011

Quilez, M., \& Vázquez, R. (2012). Aulas multigrados o el mito de la mala calidad de enseñanza en la escuela rural. Revista Iberoamericana de Educación, 2(59), 1-12. https://rieoei.org/historico/ deloslectores/5353Quilez.pdf

Revelo-Sánchez, O., Collazos-Ordóñez, C., \& Jiménez-Toledo, J. (2018). El trabajo colaborativo como estrategia didáctica para la enseñanza/aprendizaje de la programación: Una revisión sistemática de literatura. TecnoLógicas, 21(4), 115-134. http://www.scielo.org.co/scielo.php?script=sci_arttext\&pid=S0123$77992018000100008 \& \operatorname{lng}=$ en\&nrm=iso

Ricoy, C. (2006). Contribución sobre los paradigmas de investigación. Revista do Centro de Educación, 31(1), 11-22. https://www.redalyc.org/pdf/1171/117117257002.pdf

Rodríguez, G., Gil, J., \& García, E. (1996). Metodología de la investigación cualitativa. Aljibe.

Rodríguez, Y. (2004). Estrategias de enseñanza docente en escuelas multigrado. In M. Benavides (Ed.), Educación y procesos pedagógicos y equidad: Cuatro informes de investigación (pp. 131-192). Grade. http://biblioteca.clacso.edu.ar/Peru/grade/20120828112921/estrateg.pdf

Romero, H., \& Vásquez, A. (2004). Evaluación ambiental del proceso de urbanización de las cuencas del piedemonte andino de Santiago de Chile. Revista Eure, 31(94), 97-118. https://scielo.conicyt.cl/pdf/ eure/v31n94/art06.pdf

Ronquillo, E., \& Goenaga, B. (2009). Competencia comunicativa: Evolución cronológica del término y sus elementos constitutivos. Revista Humanidades Médicas, 9(1), 1-14. http://scielo.sld.cu/scielo. php?script=sci_arttext\&pid=S1727-81202009000100005 
Santos, L. (2011). Aulas multigrado y circulación de los saberes: Especificidades didácticas de la escuela rural. Revista de Curriculum y Formación de Profesorado, 15(2), 71-91. https://www.ugr.es/ recfpro/ rev152ART5.pdf

Santos, R. B. (2017). História da educação do campo no Brasil: O protagonismo dos movimentos sociais. Teias, 18(51), 210-224. https://doi.org/10.12957/teias.2017.24758

Vera, D. (2015). Resiliencia, pobreza y ruralidad. Revista Médica, 143(5), 677-678. https://scielo.conicyt.cl/ scielo.php?script $=$ sci_arttext\&pid $=$ S0034-98872015000500018

Vera, D., Osses, S., \& Schiefelbein, E. (2012). Las creencias de los profesores rurales: Una tarea pendiente para la investigación educativa. Estudios Pedagógicos, 38(1), 297-310. https://scielo.conicyt.cl/scielo. php?script=sci_arttext\&pid=S0718-07052012000100018

Vera, J., \& Peña, C. (2016). Prácticas docentes y educación multigrado en escuelas indígenas del Estado de Sonora. Revista Educación y Humanismo, 18(31), 225-240. http://revistas.unisimon.edu.co/index. php/educacion/article/view/2360

Vivas, M. (2003). La educación emocional: Conceptos fundamentales. Revista Universitaria de Investigación, 4(2), 1-22. https://www.redalyc.org/pdf/410/41040202.pdf

Vigotsky, L. (1997). El aprendizaje escolar. Aique.

Zambrano, A. (2017). Modelo de educación flexible y competencias multigrado en instituciones educativas rurales de los municipios no certificados del Valle del Cauca. Educere, 22(71), 47-59. https://www. redalyc.org/jatsRepo/356/35656002004/index.html

\section{Nota sobre autoria}

Las autoras han contribuido de manera global en la construcción del artículo.

\section{Disponibilidad de datos}

Precisamos que no es posible compartir la unidad herméutica, puesto que aún se están desarrollando y presentando nuevas investigaciones. Por lo tanto, una vez finalizado, los datos pueden estar disponibles. Sin embargo, los datos primarios que sustenta los resultados de investigación en este arículo ya se encuentran disponibles en el desarrollo del manuscrito.

\section{Cómo citar este artículo}

Freire-Contreras, P. A., Llanquín-Yaupi, G. N., Neira-Toledo, V. E., Queupumil-Huaiquinao, E. N., Riquelme-Hueichaleo, L. A., \& Arias-Ortega, K. E. (2021). Prácticas pedagógicas en aula multigrado: Principales desafíos socioeducativos en Chile. Cadernos de Pesquisa, 51, Artículo e06958. https://doi. org $/ 10.1590 / 198053147046$ 